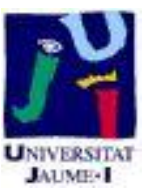

Título artículo / Títol article: Series resistance in organic bulk-heterojunction solar devices: Modulating carrier transport with fullerene electron traps

Autores / Autors

Antonio Guerrero, Teresa Ripolles-Sanchis, Pablo P. Boix, Germà Garcia-Belmonte

Revista:

Organic Electronics 13 (2012) 2326-2332

Versión / Versió:

Pre-print

Cita bibliográfica / Cita

GUERRERO, Antonio, RIPOLLÉS-SANCHIS, bibliogràfica (ISO 690): Teresa, BOIX, Pablo P., GARCÍA BELMONTE, Germà. Series resistance in organic bulkheterojunction solar devices: Modulating carrier transport with fullerene electron traps. Organic Electronics, 2012, No 13, p. 2326-2332 


\title{
Series resistance in organic bulk-heterojunction solar devices: Modulating carrier transport with fullerene electron traps
}

Antonio Guerrero, Teresa Ripolles-Sanchis, Pablo P. Boix, and Germà Garcia-Belmonte*

Photovoltaic and Optoelectronic Devices Group, Departament de Física, Universitat Jaume I, ES-12071 Castelló, Spain

*Corresponding author: G. Garcia-Belmonte, e-mail: garciag@,fca.uji.es, tel.: +34964 387548, fax: +34964 729218

\begin{abstract}
Series resistance is one of the key parameters affecting the performance of organic photovoltaic devices. Several electronic mechanisms arising from different structures within the solar cell can contribute to increasing it. We focus on the series resistance origin by altering the acceptor transport properties trough the incorporation of fullerene traps located at energies below the transporting electron levels. Indene- $\mathrm{C}_{60}$ bisadduct as acceptor molecule blended with poly(3-hexylthiophene) forms the active layer in which small amounts of [6,6]-phenyl- $\mathrm{C}_{61}$-butyric acid methyl ester have been added as trapping sites. A complete analysis of the impedance response has allowed identifying bulk transport resistive circuit elements in the high-frequency part of the spectra. Series resistance is observed to be dependent on the concentration of fullerene traps, thus indicating a connection between bulk transport processes and resistive elements. By comparing different contacts it has been discarded that outer cathode interfaces influence the series resistance experimentally extracted from impedance spectroscopy.
\end{abstract}

keywords: Photovoltaic Devices, Current-Voltage Characteristics, Series Resistance, Transport, Trapping 


\section{Introduction}

Series resistance $R_{\mathrm{S}}$ has been recognized as one of the key parameters affecting the performance of organic photovoltaic devices through reduction of the solar cell fill factor [1]. For larger series resistance values even short-circuit current might decrease. Usually $R_{\mathrm{S}}$ is estimated from the current-voltage $j-V$ curve slope at large forward voltage where the current flow is not longer limited by the internal carrier recombination but by the potential drop at $R_{\mathrm{s}}$. However it cannot be discarded that processes contributing to $R_{\mathrm{S}}$ are voltage-dependent through physical mechanisms originated at different layers or interfaces within the device. Transparent contact layers as indium tin oxide ITO, and carrier transporting interlayers of different kind could increase $R_{\mathrm{S}}$ significantly. Interfaces between the active blend layer and interlayers or metallic contacts may well add more resistance in series because of partial energy level alignment which affects optimal interface charge transfer. Finally charge carrier transport within the active layer itself could also be a source for incrementing the series resistance. The overall impact of electronic transport mechanisms is recognized to have dramatic effects when relatively thicker active layer films are used to enhance light harvesting [2]. While thinner films are able to exhibit almost $100 \%$ conversion of absorbed photons into collected carriers [3], thus indicating that transport mechanisms do not limit the achievable photocurrent [4], thicker active layer devices suffer from an incomplete collection of photogenerated charges. It is also known that in real devices the sole analysis of the $j-V$ characteristics does not help discerning which series mechanism is effectively dominating $R_{\mathrm{S}}$. Therefore the overall cell performance might be improved by quantifying operating mechanisms involved in series resistance increase.

A standard technique able to separate different resistive and capacitive contributions to the overall solar cells electrical response is impedance spectroscopy. This alternating current probing tool explores electrical mechanisms occurring within the frequency range of interest in organic solar devices, usually from $10 \mathrm{MHz}$ down to $1 \mathrm{~Hz}$. By using this analytical method we have identified circuit elements related to recombination current governing the low-frequency part of the impedance spectra $[4,5]$. Differential resistive (recombination resistance), and capacitive (chemical capacitance) processes were connected to recombination flux derivative and charge storage, respectively [6-8]. It has been also proposed that the effect of mechanisms in series is observable at high frequencies through more featured impedance spectra, which were interpreted in 
connection with electronic transport processes [9]. Very recently impedance analysis has been employed by others groups identifying a $R C$ parallel subcircuit governing the high frequency response as a combination of bulk transport resistance and geometrical capacitance $[10,11]$.

Although connecting the high-frequency impedance response to electronic transport within the solar cell bulk is a reasonable working hypothesis, no conclusive prove has been given which allows discarding additional mechanisms such as outer interfacial effects contributing to the series resistance. Deeper knowledge about the processes affecting $R_{\mathrm{S}}$ is then essential to improve power conversion efficiency and the overall cell performance. In order to progress on the $R_{\mathrm{S}}$ origin we have altered the acceptor transport properties by introducing fullerene traps located at energies below the transporting electron levels. This experimental procedure has allowed modulating the electron mobility with the consequent modification of the impedance high-frequency response. Therefore we have unambiguously identified $R C$ parallel subcircuit elements acting at high frequencies with bulk transport processes determining $R_{\mathrm{S}}$.

\section{Results and discussion}

Indene- $\mathrm{C}_{60}$ bisadduct (ICBA) $[12,13]$ has been proposed as acceptor molecule to achieve open-circuit voltage $V_{\text {oc }}$ higher than $0.8 \mathrm{~V}$ when poly(3-hexylthiophene (P3HT) is used as donor polymer. Compared to [6,6]-phenyl- $\mathrm{C}_{61}$-butyric acid methyl ester (PCBM) a shift in open-circuit voltage $\Delta V_{\mathrm{oc}} \approx 0.2 \mathrm{~V}$ is observed, in good agreement with the less negative lower-occupied molecular orbital (LUMO) level position of ICBA molecule. Cyclic voltammetry analysis of PCBM and ICBA molecules reveals a negative shift in the reduction peak of $0.17 \mathrm{~V}$ caused by the ICBA molecular LUMO position $(-3.74 \mathrm{eV})$ compared to PCBM $(-3.91 \mathrm{eV})[12]$ as illustrated in Fig. 1(a). From this energy diagram it is derived that PCBM molecules could act as trapping sites when a small amount is incorporated into the ICBA:P3HT blend, presumably altering the transport properties of electron carriers. It cannot be discarded that PCBM incorporation modifies the fullerene energetic landscape. Solar cells of structure indium tin oxide (ITO)/poly(3,4-ethylenedioxythiophene): poly(styrene sulfonic acid) (PEDOT:PSS)/ P3HT:fullerene/cathode (being cathode contact either $\mathrm{Ca} / \mathrm{Ag}$ or LiF/Al), were prepared as described in the Methods Section. In order to test the effect of the PCBM traps on the conducting properties of ICBA different fullerene acceptor composition has been tested: P3HT:ICBA, P3HT:ICBA(PCBM 0.1\%) , and 
P3HT:ICBA(PCBM 1\%) blends. Typical $j-V$ curves are shown in Fig. 1(b) for $\mathrm{Ca} / \mathrm{Ag}$ cathode contact. Photovoltaic parameters are listed in Table 1. By examining Fig. 1(b) one can realize that the incorporation of PCBM molecules largely reduces the shortcircuit current $j_{\mathrm{sc}}$. For small amount of PCBM (0.1\%) reduction in $j_{\mathrm{sc}}$ is only of $5 \%$, while PCBM larger amounts (1.0\%) induce 28\% photocurrent losses. This large reduction in $j_{\mathrm{sc}}$ might be related to lower light absorption caused by a less optimized blend morphology when PCBM molecules are included. Absorption and device external quantum efficiency $(E Q E)$ for different blend compositions are presented in Fig. 2. One can observe in Fig. 2(a) that the absorption at $\sim 600 \mathrm{~nm}$ drops around 10\% for PCBM content from $0 \%$ to $1 \%$. However EQE spectra shown in Fig. 2(b) do not follow the absorption spectral profile which peaks around $520 \mathrm{~nm}$. Instead it shows a rather flat shape between $450-600 \mathrm{~nm}$. For $1.0 \%$ of PCBM content the decrease in EQE is about $20 \%$ with respect to P3HT:ICBA-based cells. It is then observed that charge collection probability represented by $E Q E$ exhibits larger reduction in comparison to that expected from absorption spectra. A direct comparison between absorption and EQE spectra cannot be performed without caution because geometry of the absorption measurement is different to the structure of a device with electrodes. Despite this alerting point experiments suggest in this case that the sole decrease in absorption is not able to explain the large $j_{\mathrm{sc}}$ losses reported in Fig. 1 which should be then linked with mechanisms occurring after light interaction.

We move now our attention to possible series resistance influences. It is known that $j-V$ characteristics is severely distorted by effect of the series resistance, even producing a reduction in the measured $j_{\mathrm{sc}}$ with respect to the photocurrent in the case of significant $R_{\mathrm{S}}$ values [1]. Indeed voltage and current are distorted by elements in the device unrelated to the internal operation of the photoactive layer, so that $j-V$ curve is usually modeled by means of a behavior as[14]

$$
j=\frac{1}{1+R_{\mathrm{s}} / R_{\mathrm{p}}}\left\{j_{0}\left[\exp \left(\beta \frac{V_{\mathrm{app}}-j R_{\mathrm{s}}}{k_{\mathrm{B}} T / q}\right)-1\right]-\left[j_{\mathrm{ph}}-\frac{V_{\text {app }}}{R_{\mathrm{p}}}\right]\right\}
$$

Here $V_{\text {app }}$ stands for the applied voltage, and the photocurrent $j_{\mathrm{ph}}$ is reduced by the shunt resistance $R_{\mathrm{p}}$. In addition $k_{\mathrm{B}} T$ accounts for the thermal energy, $q$ is the elementary charge, and $j_{0}$ stands for the dark current. The expression in Eq. (1) includes the parameter $\beta$ accounting for the deviation from the diode ideal equation (inverse of the diode ideality factor). Interestingly the potential drop at the series 
resistance evaluated at zero bias $j_{\mathrm{sc}} R_{\mathrm{S}}$ modifies the measured $j_{\mathrm{sc}}$ with respect to the photocurrent $j_{\mathrm{ph}}$. We will next explore by using impedance spectroscopy the origin and contributing mechanisms of the series resistance.

An example of the impedance spectroscopy response is shown in Fig. 3. AC oscillating amplitude was as low as $20 \mathrm{mV}$ (rms) to maintain the linearity of the response, and the measuring frequency range was located between $1 \mathrm{MHz}$ and $100 \mathrm{~Hz}$. Impedance measurements have been performed at different applied voltages under 1 sun illumination. The comparison between light and dark responses of P3HT:PCBM solar devices was addressed in a previous work [4] concluding that a master $j-V$ curve is obtained after correcting the ohmic voltage drop at the series resistance. In the case of relatively low series resistance values this allows studying exclusively light impedance responses to obtain relevant cell parameters. It is observed in Fig. 3 that the impedance spectra comprise two visually distinguishable parts. At lower frequencies a large $R C$ subcircuit (represented by a semicircle in $Z^{\prime}-Z^{\prime \prime}$ plot) dominates. As explained in previous works this part is related to the chemical capacitance $C_{\mu}$ (connected to carrier storage) and recombination resistance $R_{\text {rec }}$ (derivative of the carrier recombination flux) response [6-8]. $R_{\text {rec }}$ and $C_{\mu}$ as a function of the applied voltage are drawn in Fig. 4. As observed exactly the same behavior is found with small differences presumably related to variation of fullerene energetics. By examining Fig. 4(b) one can observe a negative voltage shift in the capacitance of 1.0\% PCBM content devices with respect to that of P3HT:ICBA cells. Such voltage shift is related to lower lying energy levels introduced by PCBM molecules after interaction with ICBA. If this energy shift is corrected a collapse of the $R_{\text {rec }}-V_{\text {app }}$ plots in Fig. 4(a) are obtained. Similar $R_{\text {rec }}$ behavior entails that the PCBM incorporation does not alter significantly recombination mechanisms [15].

In this work we will focus our attention on electronic processes affecting the highfrequency part of the impedance spectra. In Fig. 3(b) an additional semicircle $R_{1} C_{1}$ is clearly distinguishable at larger forward voltages $(0.7-0.8 \mathrm{~V})$ and higher frequencies, which is connected here to transport effects as discussed later. The overall impedance response also includes a resistance connected in series $R_{0} \approx 2 \Omega \mathrm{cm}^{2}$ that is attributed to ITO sheet and wires contributions, and results practically voltage-independent. This general trend is always reproduced despite modifications introduced either in the blend composition, or cathode contact. The circuit model used in fitting is drawn in Fig. 3(c). Alternative circuit models as that comprising two $R C$ subcircuits in series have been 
checked producing in this case slightly poor fittings (see Supplementary data file). For the relevant voltage range $(<0.7 \mathrm{~V})$ parameter errors are always below $5 \%$, what allows omitting error bars in Fig. 4 and 5 because they are smaller than the dot size.

A close look at the variation of $R_{1} C_{1}$ high-frequency subcircuit elements with the applied voltage is plotted in Fig. 5. When PCBM trap molecules are not incorporated into the active layer $R_{1}$ exhibits low values $\left(30-10 \Omega \mathrm{cm}^{2}\right)$ irrespective of the cathode contact used. If lower amounts of PCBM molecules are inserted (0.1\%) slightly higher $R_{1}$ values are encountered. The response changes drastically when the PCBM content amounts $1.0 \%$ of acceptor molecules. In this last case a large $R_{1}$ contribution is found that steeply decreases from $\sim 100 \Omega \mathrm{cm}^{2}$ at low voltages down to $10 \Omega \mathrm{cm}^{2}$ for voltages in excess of $V_{\mathrm{oc}}$. This kind of behavior observed for the transport-related resistance $R_{1}$ can be understood in terms of a trap-modulated conductivity mechanism as follows: acceptor ICBA LUMO levels form a sort of transporting band, while PCBM molecules behave as trapping sites for negative polarons (electrons) as schematically drown in Fig. 1(a). When the amount of PCBM is low $R_{1}$ slightly decreases with the applied voltage because trapping effects are very limited. For larger PCBM concentration the probability of electron trapping increases then reducing the carrier mobility, and consequently the measured resistance. The applied voltage also has an influence on $R_{1}$ by modifying the acceptor LUMO level occupancy. For low applied bias PCBM trap sites are not populated because of a deeper position of the electron Fermi level. Mobile electrons can then easily undergo trapping producing a decrease in the carrier conductivity. As the electron Fermi level rises following the increment in forward voltage PCBM trap sites are eventually occupied losing their influence on $R_{1}$. Interestingly $R_{1}$ exhibits values that are fullerene content-independent at larger forward voltage as expected when trapping sites are no longer active. The above mentioned conduction mechanism is highly sensitive to the kinetics of trapping/release of trapped electrons at PCBM molecules. While the inclusion of fast traps does not modify the carrier conductivity [16], slow trapping would have large influence on the transport mechanisms producing conductivity changes. It is worth noting that similarity of $R_{1}$ values at forward bias allows discarding morphology effects as originating the $R_{1}$ differences observed. On the other hand the capacitive element $C_{1}$ exhibits a rather constant value which is related to geometrical and dielectric mechanisms. Variations of $C_{1}$ observed among samples might be caused by a combination of several factors: differences in active layer thickness, changes in blend permittivity by PCBM 
incorporation, or influence of contact capacitive effects could all be behind the actual $C_{1}$ value. Nevertheless $C_{1}$ differences are limited by a factor of 2 .

We now return to the issue concerning the outer interface contribution on the measured series resistance, in contrast to the transport-originated mechanism discussed previously. To discern between contact from bulk origins two different cathode contacts have been deposited, namely $\mathrm{Ca}$ and $\mathrm{LiF} / \mathrm{Al}$. It is observed in Fig. 5 that $R_{1}$ behaves in a way that appears independent of the cathode contact used. For 1.0\% PCBM fullerene content the same increment in $R_{1}$ is experimentally found. It is worth noticing the poor performance of cells contacted with $\mathrm{LiF} / \mathrm{Al}$ (see Table 1). This is presumably caused by interactions at the cathode interface that impede a proper charge extraction. Despite this evident limitation at the cathode interface, bulk transport properties exhibit similar behavior than that obtained for devices contacted with $\mathrm{Ca}$. These experimental trends reinforce our previous idea pointing to transport rather than interface origin for the highfrequency impedance response. However it cannot be completely excluded the possibility that anode interface or PEDOT:PSS layer contribute to $R_{\mathrm{S}}$ in the case of lower PCBM content.

When the combined contribution of $R_{1}$ and $R_{0}$ is connected in series $R_{\mathrm{s}}=R_{1}+R_{0}$, it is feasible to calculate the voltage responsible for the photovoltaic effect as

$$
V_{F}=V_{\text {app }}-j R_{\mathrm{s}}
$$

in accordance to Eq. (1). We have explained in previous papers how $V_{F}$ is in fact originated by the Fermi level splitting corresponding to separate carriers [5]. The actually measured $j-V$ characteristics exhibits then a distortion in the voltage axis with respect to the Fermi level splitting produced by the series resistance potential drop [4]. This voltage axis correction allows for an estimation of the achievable power conversion efficiency in the case of complete elimination of the series resistance effect as listed in Table 1 for Ca cathode contact. After correction of $R_{\mathrm{S}}$ effect PCE increases as expected but it does not reach a similar value because differences in $j_{\mathrm{sc}}$ caused by absorption losses, and reduction in $V_{\text {oc }}(\sim 70 \mathrm{meV})$ originated by fullerene energetics variation are still present. The approach implied by Eq. (2) should be taken with caution because the series resistance potential drop is only a useful approximation of a rather complex picture concerning the Fermi level distribution across the active layer thickness [17]. In general terms transport mechanisms are coupled with charge storage and recombination (represented by $C_{\mu}$ and $R_{\text {rec }}$ ) in a transmission line circuit model [9], 
instead of the circuit comprising lumped elements as in Fig. 3(c). Only in those cases in which the transport resistance is significantly lower than $R_{\text {rec }}$ the application of the correction in Eq. (2) is fully justified. A detailed analysis including transmission line circuits will be addressed in forthcoming works.

\section{Conclusion}

We have focused on carrier transport effects by means of a simple equivalent circuit model directly derived from the inspection of impedance spectra corresponding to P3HT:ICBA bulk-heterojunction solar cells. By incorporating PCBM molecules into the blend electron traps have been created which modulate the electron conductivity. Consequently the high-frequency part of the impedance spectra undergo significant alteration in its resistive component. It has been demonstrated by using different cathode contacts that high-frequency resistances only depend on the active layer blend composition, and not on the outer contact structure. Impedance spectroscopy appears then as a powerful tool applicable to evaluate losing mechanisms in complete and operative organic solar cells.

\section{Methods}

Device fabrication.

P3HT (Aldrich), ICBA (Luminescence Technology Corp.), $\mathrm{PC}_{60} \mathrm{BM}$ (Nano-C, 99.5 \%), PEDOT:PSS (CLEVIOS P AI 4083), o-dichlorobenzene (Aldrich, $99.9 \%$ ), Ca (Aldrich, $99.995 \%$ ), silver (Aldrich, $99.99 \%$ ), LiF (Aldrich, $99.995 \%$ ) and Al (Kurt J. Lesker, $99.999 \%$ ) were used as received without further purification. All manipulations were carried out in a glovebox under a nitrogen atmosphere unless otherwise stated. P3HT:fullerenes blends were prepared from dry o-dichlorobenzene $(1: 0.8,17 \mathrm{mg} / \mathrm{ml})$ and were stirred at R.T. for $2 \mathrm{~h}$ before sample preparation. The fullerene acting as an electron trap $\left(\mathrm{PC}_{60} \mathrm{BM}\right)$ when added provided a concentration of either $0.1 \%$ or $1 \%$ by weight. Polymer solar cells were fabricated with a standard sandwich structure of ITO/PEDOT:PSS/P3HT:Fullerenes/Cathode, and $9 \mathrm{~mm}^{2}$ of active area (Cathode $=\mathrm{Ca} /$ $\mathrm{Ag}$ or LiF/Al). PEDOT:PSS was spin coated in air at $5500 \mathrm{rpm}$ for 30 seconds onto an ITO coated glass substrate $(10 \Omega / \mathrm{sq})$, film thickness of $\sim 35 \mathrm{~nm}$. The substrates were heated at $120{ }^{\circ} \mathrm{C}$ for $10 \mathrm{~min}$ to remove traces of water and were transferred to a glovebox equipped with a thermal evaporator. The P3HT:fullerene layer was deposited at speeds of $1200 \mathrm{rpm}$ (thickness was about $110 \mathrm{~nm}$ ) for 60 seconds to provide a dry 
film. At this point, samples were thermally annealed at $150{ }^{\circ} \mathrm{C}$ for $10 \mathrm{~min}$. Evaporation was carried out at a base pressure of $3 \times 10^{-6}$ mbar with the cathode under study either $\mathrm{Ca} / \mathrm{Ag}(5 / 100 \mathrm{~nm})$ or $\mathrm{LiF} / \mathrm{Al}(1 / 100 \mathrm{~nm})$. Devices were sealed with a photoresin and a glass microscopy slide followed by exposure under UV light. Samples were then taken out of the glovebox for device characterization.

\section{Device characterization.}

Current density-voltage and impedance spectroscopy measurements were carried out by illumination with a $1.5 \mathrm{G}$ illumination source $\left(1000 \mathrm{~W} \mathrm{~m}^{-2}\right)$ using an Abet Sun 2000 Solar Simulator. The light intensity was adjusted with a calibrated Si solar cell. Impedance spectroscopy measurements were performed with Autolab PGSTAT-30 equipped with a frequency analyzer module, and was recorded by applying a small voltage perturbation (20 mV rms). Measurements were carried out at 1 sun light intensity at different DC bias voltage sweeping frequencies from $1 \mathrm{MHz}$ down to 100 Hz. UV/Vis data was obtained from films using a Cary 300 Bio Spectrophotometer. External Quantum Efficiency (EQE) measurements were performed using a $150 \mathrm{~W}$ Xe lamp coupled with a monochromator controlled by a computer. The light intensity was measured using an optical power meter 70310 from Oriel Instruments where a Si photodiode was used to calibrate the system.

\section{Supplementary data}

Details of impedance fitting procedure and comparison between different equivalent circuits are provided as Supplementary dada file.

\section{Acknowledgement}

We thank financial support from Ministerio de Educacion y Ciencia under project HOPE CSD2007-00007 (Consolider-Ingenio 2010), Generalitat Valenciana (Prometeo/2009/058, ACOMP/2009/056, ACOMP/2009/095, and ISIC/2012/008 Institute of Nanotechnologies for Clean Energies), and FP7 European project ORION (Large CP-IP 229036-2). 


\section{References}

[1] R.A. Street, K.W. Song, S.R. Cowan, Influence of Series Resistance on the Photocurrent Analysis of Organic Solar Cells, Organic Electronics, 12 (2011) 244-248. [2] S.R. Cowan, N. Banerji, W.L. Leong, A.J. Heeger, Charge Formation, Recombination, and Sweep-Out Dynamics in Organic Solar Cells, Advanced Functional Materials, 22 (2012) 1116-1128.

[3] S.H. Park, A. Roy, S. Beaupré, S. Cho, N. Coates, J.S. Moon, D. Moses, M. Leclerc, K. Lee, A.J. Heeger, Bulk heterojunction solar cells with internal quantum efficiency approaching 100\%, Nature Photonics, 3 (2009) 297 - 302.

[4] P.P. Boix, A. Guerrero, L.F. Marchesi, G. Garcia-Belmonte, J. Bisquert, CurrentVoltage Characteristics of Bulk Heterojunction Organic Solar Cells: Connection Between Light and Dark Curves, Advanced Energy Materials, 1 (2011) 1073-1078. [5] J. Bisquert, G. Garcia-Belmonte, On voltage, photovoltage and photocurrent in bulk heterojunction organic solar cells, J. Phys. Chem. Lett., 2 (2011) 1950-1964.

[6] G. Garcia-Belmonte, P.P. Boix, J. Bisquert, M. Sessolo, H.J. Bolink, Simultaneous determination of carrier lifetime and electron density-of-states in P3HT:PCBM organic solar cells under illumination by impedance spectroscopy, Sol. Energy Mater. Sol. Cells, 94 (2010) 366-375.

[7] W.L. Leong, S.R. Cowan, A.J. Heeger, Differential Resistance Analysis of Charge Carrier Losses in Organic Bulk Heterojunction Solar Cells: Observing the Transition from Bimolecular to Trap-Assisted Recombination and Quantifying the Order of Recombination, Advanced Energy Materials, 1 (2011) 517-522.

[8] Y. Zhang, X.-D. Dang, C. Kim, T.-Q. Nguyen, Effect of Charge Recombination on the Fill Factor of Small Molecule Bulk Heterojunction Solar Cells, Advanced Energy Materials, 1 (2011) 610-617.

[9] G. Garcia-Belmonte, A. Munar, E.M. Barea, J. Bisquert, I. Ugarte, R. Pacios, Charge carrier mobility and lifetime of organic bulk heterojunctions analyzed by impedance spectroscopy, Organic Electronics, 9 (2008) 847-851.

[10] B.J. Leever, C.A. Bailey, T.J. Marks, M.C. Hersam, M.F. Durstock, In Situ Characterization of Lifetime and Morphology in Operating Bulk Heterojunction Organic Photovoltaic Devices by Impedance Spectroscopy, Advanced Energy Materials, 2 (2012) 120-128.

[11] G. Perrier, R. deBettignies, S. Berson, N. Lemaître, S. Guillerez, Impedance spectrometry of optimized standard and inverted P3HT-PCBM organic solarcells, Sol. Energy Mater. Sol. Cells, 101 (2012) 210-216.

[12] Y. He, H.-Y. Chen, J. Hou, Y. Li, Indene-C60 Bisadduct: a New Acceptor for High-Performance Polymer Solar Cells, Journal of American Chemical Society, 132 (2010) 1377-1382. 
[13] G. Zhao, Y. Hi, Y. Li, 6.5\% Efficiency of Polymer Solar Cells Based on poly(3hexylthiophene) and Indene-C 60 Bisadduct by Device Optimization, Adv. Mat., 22 (2010) 4355-4358.

[14] S.M. Sze, Physics of Semiconductor Devices, 2nd ed., John Wiley and Sons, New York, 1981.

[15] A. Guerrero, L.F. Marchesi, P.P. Boix, J. Bisquert, G. Garcia-Belmonte, Recombination in Organic Bulk Heterojunction Solar Cells: Small Dependence of Interfacial Charge Transfer Kinetics on Fullerene Affinity, J. Phys. Chem. Lett., 3 (2012) 1386-1392.

[16] J. Bisquert, Interpretation of electron diffusion coefficient in organic and inorganic semiconductors with broad distribution of states, Phys. Chem. Chem. Phys., 10 (2008) 3175-3194.

[17] A. Pitarch, G. Garcia-Belmonte, I. Mora-Seró, J. Bisquert, Electrochemical impedance spectra for the complete equivalent circuit of diffusion and reaction under steady-state recombination current, Phys. Chem. Chem. Phys., 6 (2004) 2983-2988. 


\section{Table captions}

\section{Table I}

Photovoltaic parameters corresponding to P3HT:fullerene solar cells with different cathode metal. ${ }^{*}$ Corrected fill factor FF and power conversion efficiency PCE by elimination of the series resistance potential drop in the case of Ca contact

\begin{tabular}{|l|l|r|r|r|r|r|r|}
\hline \multicolumn{2}{|l|}{} & $\begin{array}{c}j_{\mathrm{sc}} \\
\left(\mathrm{mA} \mathrm{cm}^{-2}\right)\end{array}$ & $\begin{array}{c}V_{\mathrm{oc}} \\
(\mathrm{mV})\end{array}$ & $F F$ & $\begin{array}{c}\text { PCE } \\
(\%)\end{array}$ & $F F^{*}$ & $\begin{array}{c}\text { PCE* } \\
(\%)\end{array}$ \\
\hline \multirow{2}{*}{$\begin{array}{l}\text { P3HT:ICBA } \\
\text { cathode Ca/Ag }\end{array}$} & no PCBM & 8.0 & 872 & 0.60 & 4.2 & 0.80 & 5.5 \\
\cline { 2 - 8 } & $0.1 \%$ PCBM & 7.7 & 877 & 0.54 & 3.7 & 0.74 & 5.0 \\
\cline { 2 - 8 } & $1.0 \%$ PCBM & 5.7 & 805 & 0.54 & 2.9 & 0.69 & 3.5 \\
\hline \multirow{2}{*}{$\begin{array}{l}\text { P3HT:ICBA } \\
\text { LiF/A1 }\end{array}$} & no PCBM & 6.2 & 735 & 0.59 & 2.7 & & \\
\cline { 2 - 9 } & $0.1 \%$ PCBM & 5.9 & 681 & 0.47 & 1.9 & & \\
\cline { 2 - 8 } & $1.0 \%$ PCBM & 3.4 & 662 & 0.55 & 1.2 & & \\
\hline
\end{tabular}




\section{Figures}
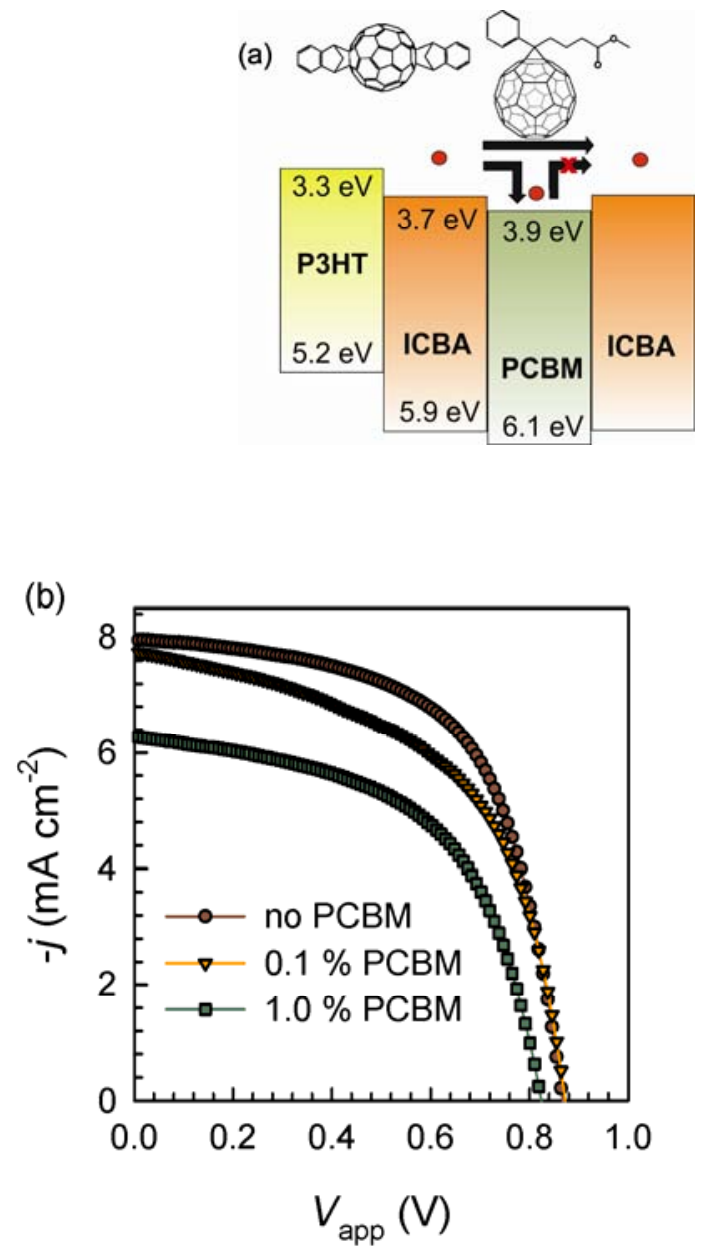

Fig. 1

(a) Energy level diagram of the active polymer and fullerene molecules used. Note that PCBM LUMO level is situated at more negative energies with respect to ICBA LUMO, then acting as trapping sites for electrons moving trough ICBA molecules. (b) $j-V$ curves corresponding to P3HT:ICBA(no PCBM), P3HT:ICBA(PCBM 0.1\%), and P3HT:ICBA(PCBM 1.0\%) blends for Ca cathode contact. 
(a)

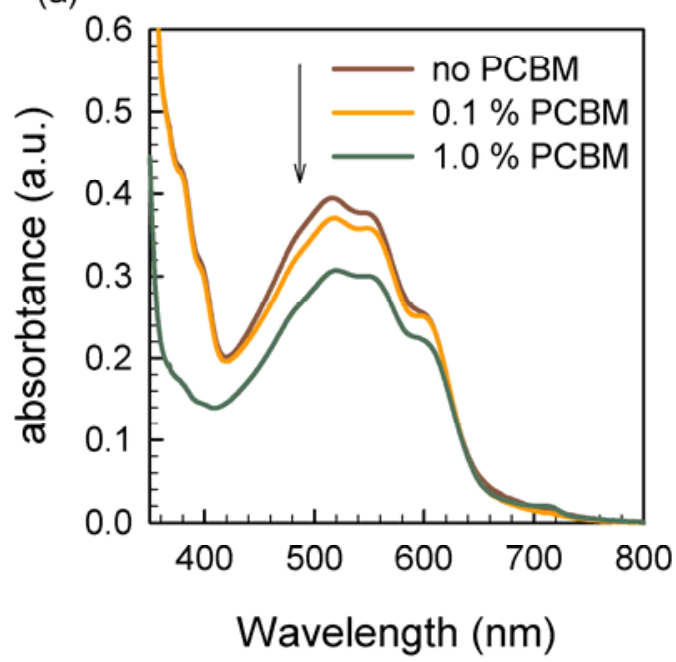

(b)

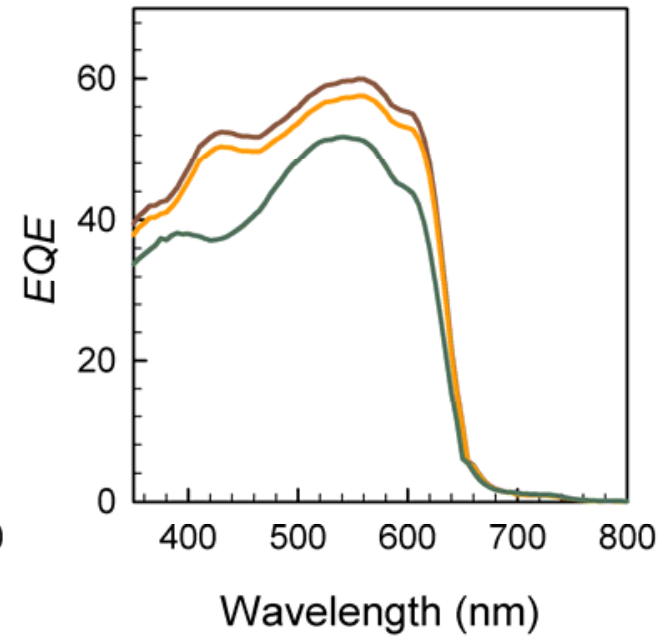

Fig. 2

(a) Absorption spectra of P3HT:ICBA films incorporating different content of PCBM molecules as indicated. (b) External quantum efficiency spectra of P3HT:ICBA devices incorporating different content of PCBM molecules. 

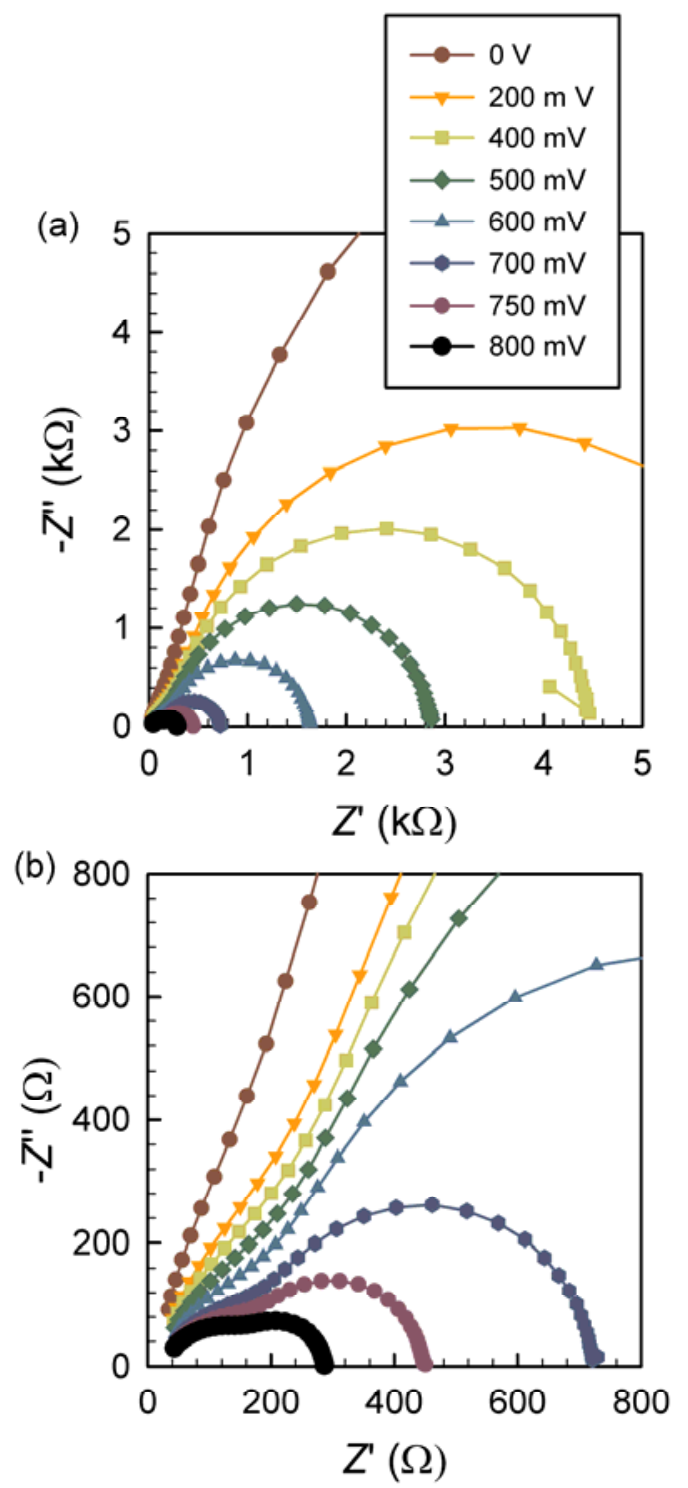

(c)

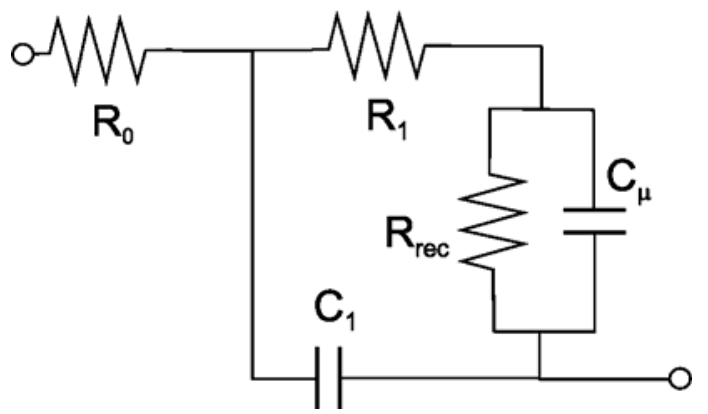

Fig. 3

(a) Impedance spectra for different applied voltage values as indicated obtained for the 
structure ITO/PEDOT:PSS/P3HT:ICBA/Ca under 1 sun illumination intensity. (b) Detail of the impedance spectra in Fig. 3(a) showing the high-frequency $R_{1} C_{1}$ contribution (left) and the main $R_{\text {rec }} C_{\mu}$ arc (right). (c) Simple equivalent circuit using in data fitting.
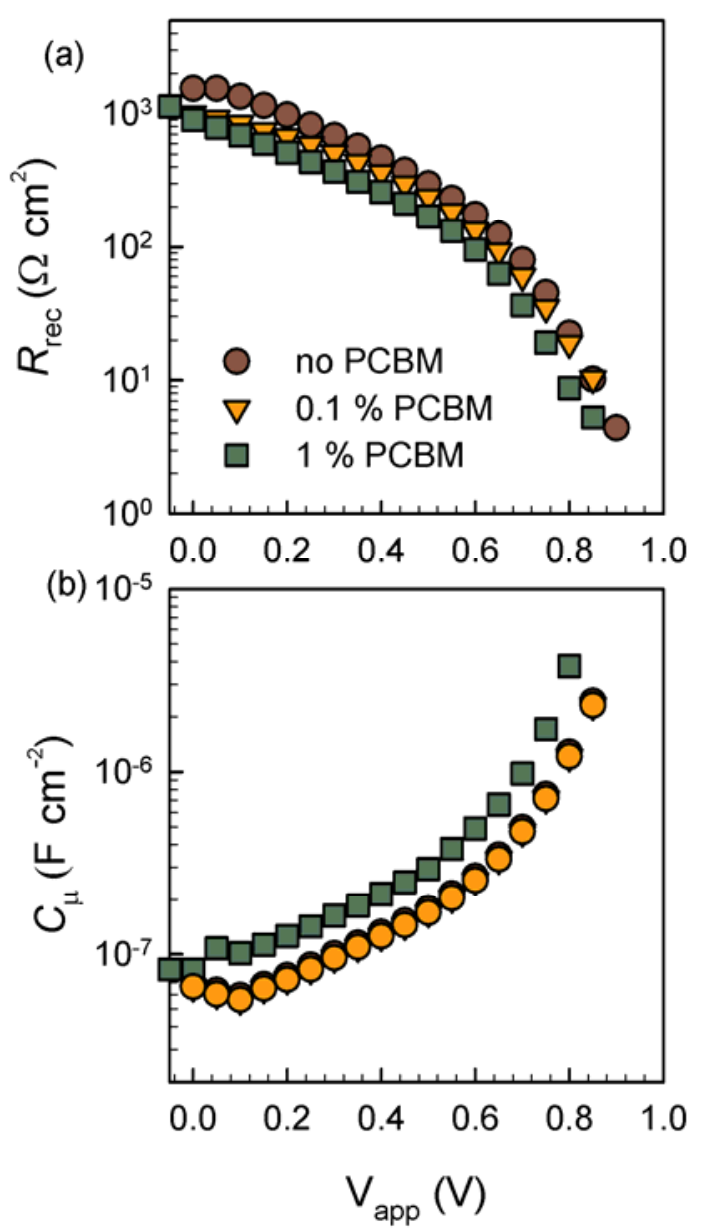

\section{Fig.4}

(a) Recombination resistance and (b) chemical capacitance obtained from fitting of impedance spectra using the equivalent circuit of Fig. 3(c). Error bars calculated from fitting smaller than the dot size. 

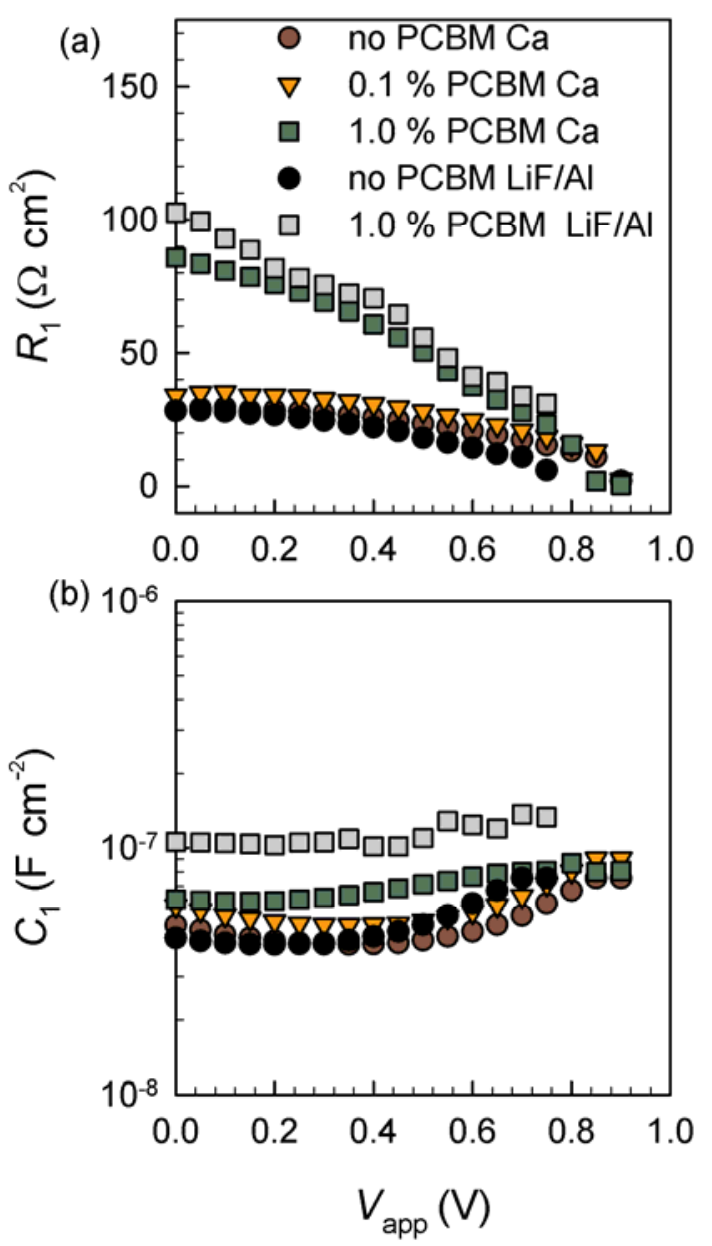

Fig.5

(a) Bulk transport resistance and (b) geometrical, dielectric capacitance obtained from fitting of impedance spectra using the equivalent circuit of Fig. 3(c). Two different cathode contacts are compared: $\mathrm{Ca}$ and $\mathrm{LiF} / \mathrm{Al}$. Error bars calculated from fitting smaller than the dot size.

\section{Graphical Abstract}

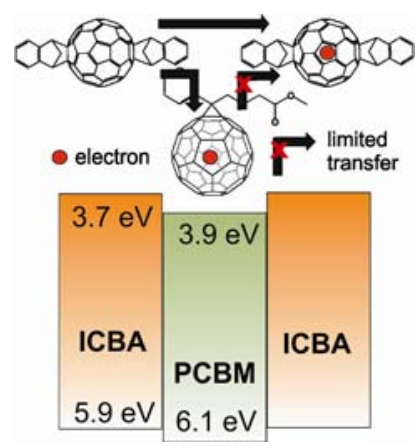


Supplementary data

\title{
Series resistance in organic bulk-heterojunction solar devices: Modulating carrier transport with fullerene electron traps
}

\author{
Antonio Guerrero, Teresa Ripolles-Sanchis, Pablo P. Boix, and Germà Garcia-Belmonte*
}

Photovoltaic and Optoelectronic Devices Group, Departament de Física, Universitat

Jaume I, ES-12071 Castelló, Spain

\section{Comparison between alternative equivalent circuit models}

A different equivalent circuit model to that presented in the main paper was evaluated. Figure SI1 and Fig. SI2 show fitting examples using both equivalent circuits. In these Figures the equivalent circuits are shown with the experimental and fitting values. Both capacitance vs frequency spectra and impedance plot show that the equivalent circuit presented in the main text provides a better fit. The goodness of the fitting is very similar for both equivalent circuits as can be observed from the error values shown in Table SI1. Only at high voltages values obtained for the alternative equivalent circuit (Fig. SI2a) is slightly higher. Values extracted for $R_{\text {rec }}, C \mu, R_{1}$ and $C_{1}$ are slightly different when the different equivalent circuits are used (see Figure SI3), however, the overall trends are the same.

Figure SI1: Example of agreement between experimental data and fitting results for a cell fabricated with P3HT:ICBA and 1\% of PCBM trap. a) Equivalent circuit used for the fitting and (in this work) b) capacitance spectra c) Impedance spectra. Measurement was taken at 1 sun light intensity and using a DC bias of $0.7 \mathrm{~V}$. 
a)

b)
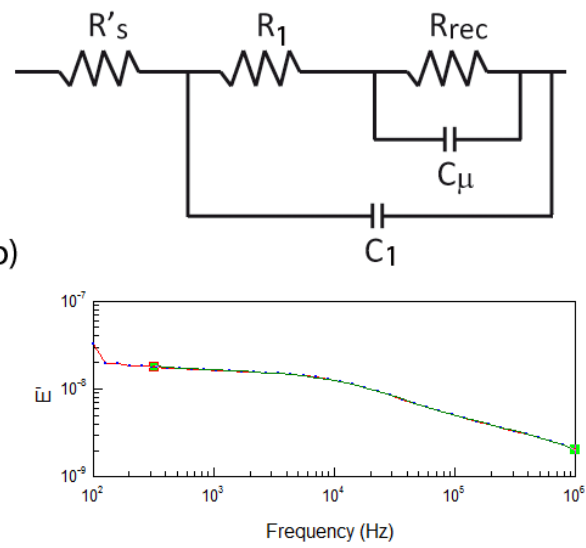

c)

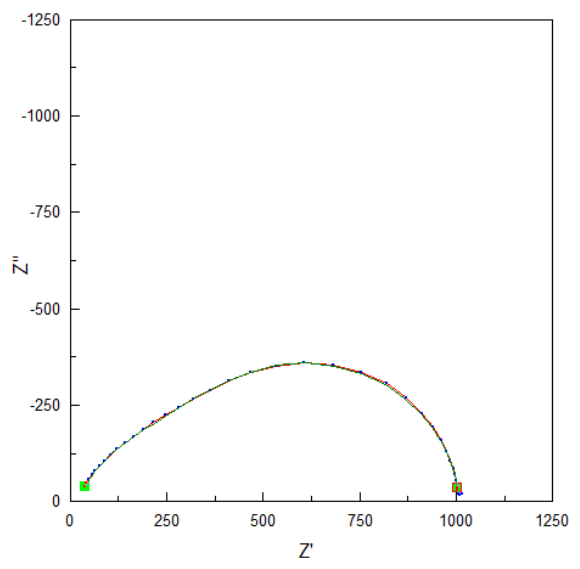

Figure SI2: Example of agreement between experimental data and fitting results for a cell fabricated with P3HT:ICBA and 1\% of PCBM trap. a) Alternative equivalent circuit that could be used b) capacitance spectra c) Impedance spectra. Measurement was taken at 1 sun light intensity and using a DC bias of $0.7 \mathrm{~V}$.

a)

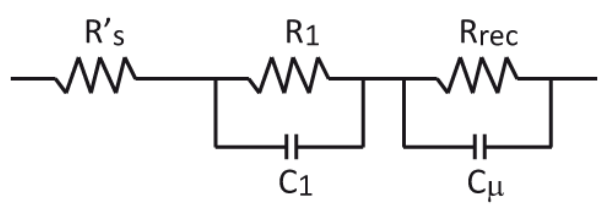

b)

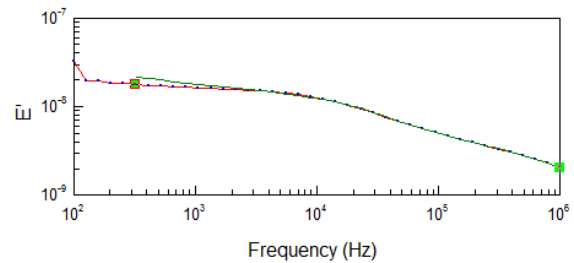

c)

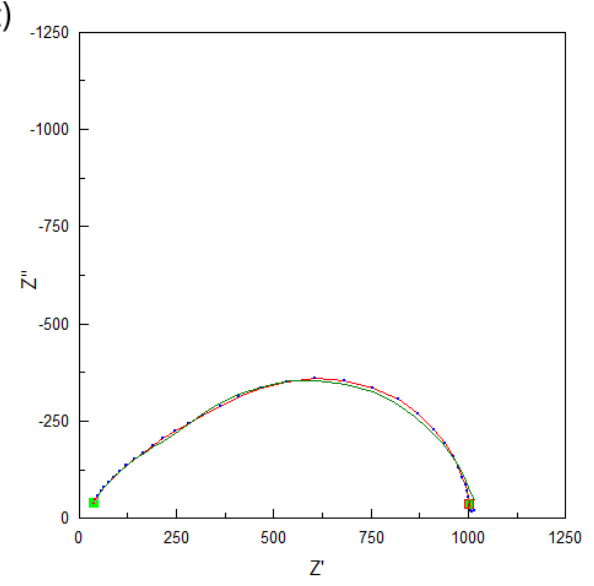


Figure SI3: Fitting results for a cell fabricated with P3HT:ICBA and 1\% of PCBM trap using the equivalent circuits shown in Fig. SIla and Fig. SI2a.
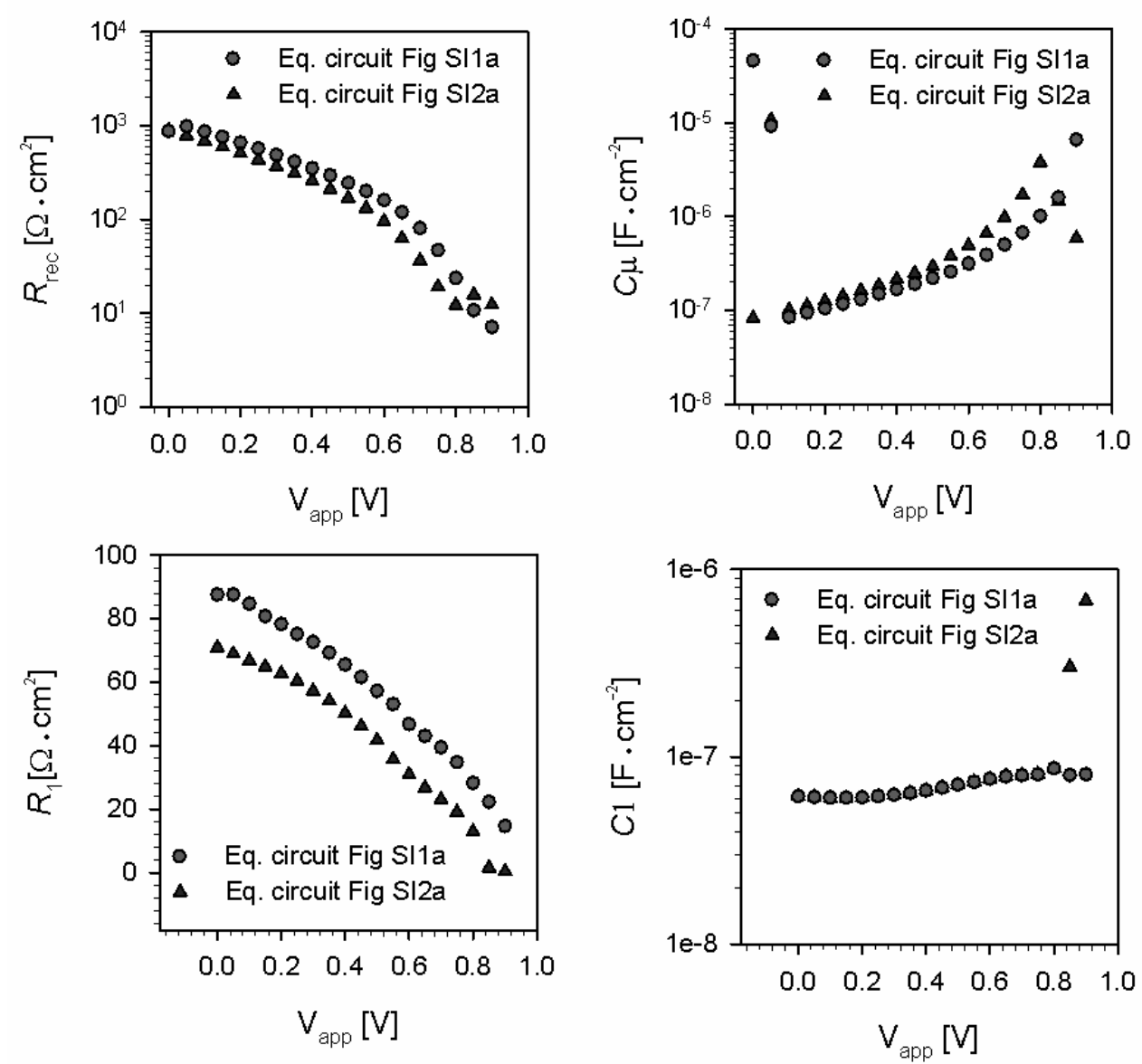
Table SI1: Errors associated to the fitted values shown in Figure SI3 for both equivalent circuits used.

\begin{tabular}{|c|c|c|c|c|c|c|c|c|c|c|}
\hline \multirow[b]{2}{*}{$\begin{array}{r}\text { Voltage } \\
\text { [V] }\end{array}$} & \multicolumn{5}{|c|}{ Main text Eq. Circuit (Fig. SI1a) } & \multicolumn{5}{|c|}{ Alternative Eq. Circuit (Fig. SI2a) } \\
\hline & $\begin{array}{r}\text { Rs } \\
\text { Error } \\
\text { [\%] }\end{array}$ & $\begin{array}{l}\text { R1 } \\
\text { Error } \\
\text { [\%] }\end{array}$ & $\begin{array}{l}\text { C1 } \\
\text { Error } \\
{[\%]} \\
\end{array}$ & $\begin{array}{l}\text { Rrec } \\
\text { Error } \\
\text { [\%] } \\
\end{array}$ & $\begin{array}{l}\mathrm{C} \mu \\
\text { Error } \\
{[\%]}\end{array}$ & $\begin{array}{r}\text { Rs } \\
\text { Error } \\
\text { [\%] }\end{array}$ & $\begin{array}{r}\text { R1 } \\
\text { Error } \\
\text { [\%] }\end{array}$ & $\begin{array}{l}\text { C1 } \\
\text { Error } \\
{[\%]} \\
\end{array}$ & $\begin{array}{l}\text { Rrec } \\
\text { Error } \\
\text { [\%] }\end{array}$ & $\begin{array}{l}\quad \mathrm{C} \mu \\
\text { Error } \\
{[\%]}\end{array}$ \\
\hline 0 & 4 & 2 & $<1$ & 2 & 4 & 3 & 1 & 3 & 5 & 6 \\
\hline 0.05 & 4 & 2 & $<1$ & 2 & 4 & 3 & 1 & 3 & 4 & 5 \\
\hline 0.1 & 4 & 2 & $<1$ & 2 & 5 & 3 & 1 & 3 & 4 & 5 \\
\hline 0.15 & 4 & 2 & $<1$ & 2 & 5 & 3 & 1 & 3 & 4 & 5 \\
\hline 0.2 & 4 & 2 & $<1$ & 2 & 5 & 3 & 1 & 3 & 4 & 5 \\
\hline 0.25 & 4 & 2 & $<1$ & 1 & 5 & 3 & 1 & 4 & 4 & 5 \\
\hline 0.3 & 4 & 2 & $<1$ & 1 & 5 & 3 & 1 & 4 & 4 & 5 \\
\hline 0.35 & 3 & 1 & $<1$ & 1 & 5 & 3 & 1 & 4 & 3 & 5 \\
\hline 0.4 & 3 & 1 & $<1$ & 1 & 6 & 3 & 1 & 4 & 4 & 6 \\
\hline 0.45 & 3 & 1 & $<1$ & 1 & 6 & 3 & 1 & 5 & 4 & 6 \\
\hline 0.5 & 3 & 1 & $<1$ & 1 & 6 & 3 & 1 & 5 & 4 & 7 \\
\hline 0.55 & 3 & 1 & $<1$ & 1 & 6 & 3 & 2 & 7 & 5 & 8 \\
\hline 0.6 & 3 & 1 & $<1$ & 1 & 7 & 3 & 2 & 7 & 5 & 9 \\
\hline 0.65 & 3 & 1 & $<1$ & 1 & 7 & 3 & 3 & 8 & 5 & 10 \\
\hline 0.7 & 2 & 1 & $<1$ & 1 & 7 & 3 & 4 & 9 & 6 & 10 \\
\hline 0.75 & 2 & 2 & 2 & 11 & 11 & 2 & 8 & 12 & 7 & 11 \\
\hline 0.8 & 1 & 1 & 2 & 10 & 8 & 2 & 20 & 14 & 18 & 11 \\
\hline 0.85 & 1 & 1 & 4 & 14 & 8 & 3 & 9 & 7 & 91 & 123 \\
\hline 0.9 & 1 & 2 & 10 & 26 & 11 & 1 & 1 & 3 & 26 & 60 \\
\hline
\end{tabular}

